\title{
AN EXPERIMENTAL STUDY OF THE COMPRESSION PROPERTIES OF POLYURETHANE-BASED WARP-KNITTED SPACER FABRIC COMPOSITES
}

\author{
Si Chen, Xue-pei Zhang, Hong-xia Chen, Xiao-ping Gao \\ College of Light Industry and Textile, Inner Mongolia University of Technology, Hohhot, China \\ Correspondence to: Si Chen email: ansn9119@126.com
}

\begin{abstract}
:
The present work has reported the compression properties of polyurethane-based warp-knitted spacer fabric composites (PWSF). In order to investigate the effect of structural parameters of fabric on the compression performance of composites, a series of warp-knitted spacer fabrics (WSF) with different structural parameters including spacer yarn inclination angle, thickness, fineness of spacer yarns, and outer layer structure have been involved. The produced composites have been characterized for compression properties. The energy-absorption performance during the compression process has been determined as a function of the efficiency and the compression stress obtained from compression tests. The results show that the composites based on spacer fabrics having smaller spacer yarns inclination angle, higher fabric thickness, finer spacer yarn, and larger mesh in outer layers perform better with respect to energy-absorption properties at lower stress level, whereas at higher stress level, the best energy-absorption abilities are obtained in case of spacer fabrics constructed of larger spacer yarn inclination angle, lower fabric thickness, coarser spacer yarn, and smaller mesh in surface layers.
\end{abstract}

\section{Keywords:}

Compression properties, Energy-absorption capacities, Structural parameters, Warp-knitted spacer fabrics

\section{Introduction}

Recently, textile fabrics are commonly used as alternative low-cost reinforcement for structural applications, such as automotive, packaging, building products, furniture, and consumer goods [1]. The various applications are demanding complex-shaped fabrics to meet the requirements of such domains [2]. Warp-knitted spacer fabrics (WSF) are one of the complex-shaped 3D constructions composed of two separate fabric layers connected by spacer yarns. The spacer yarns have different inclination angles depending on the different lapping movements of guide bars. And the thickness of spacer fabrics can also be designed. Moreover, WSF composites exhibit greater drapability and compression resistance capacities as compared to other textile-reinforced composites. All of these advantages make WSF to obtain great potential to be used for the reinforcement of composites.

In some of the published studies, the compression behaviors of composites reinforced by knitted spacer fabrics have been examined [3-7]. These composites were produced based on an unsaturated resin. It can be found in these studies that the structural parameters of spacer fabrics have obvious influences on the compression properties of these composites. Also, a type of sandwich composite panels based on WSF, which have a considerably lightweight core, has been fabricated by using modified vacuum assisted resin transfer molding (VARTM) technology [8]. This study shows that the panels with higher cross-thread density and finer yarns exhibit high facing bending stress and core shear stiffness. And coarser cross-thread used in the core results in better compression responses. Recently,
Vuure [9] has developed a unit-cell model of core properties of composite panels based on spacer fabrics, and the finite element method calculations of the compression responses of these novel composites have been performed.

The present work reports the compression properties of polyurethane-based WSF composites for cushioning applications. With an attempt to discuss the effect of fabric structural parameters on compression properties of the novel composites, a series of WSF with different parameters (such as spacer yarn inclination angle, thickness, diameter of spacer yarn, and outer layer structure) were fabricated on a doubleneedle-bar warp knitting machine of E18. It is expected that a regular pattern for tailoring WSF composites with favorable compression responses could be found from this study.

\section{Experiment part}

\subsection{Samples}

\section{Warp-knitted spacer fabrics}

Eight WSF with different structural parameters were involved in this study. They were produced on a double-needle-bar Raschel warp knitting machine of E18 (Wuyang CO. LTD, Jiangsu, China). The PET monofilament of $0.2 \mathrm{~mm}(0.16 \mathrm{~mm})$ in diameter was used as spacer yarns, and 300D/96F PET multifilament was used to knit the surface layers of fabrics. Three different structures, that is, Chain+Inlay, Rhombic Mesh, and Hexagonal Mesh, were involved for knitting surface layers. 
The chain notations for each outer layer structure are listed in Table 1. Three types of spacer yarns were used to connect the two outer layers with different inclination angles. The details of spacer yarns are presented in Table 2. By considering the different types of spacer yarns, different thickness, and different outer layer structures, eight WSF were produced. The structural parameters of these WSF are shown in Table 3. The front elevation and right view of WSF1, which is chosen as the representative of eight samples, are provided in Figure 1.

\section{Preparation of the composites}

The warp-knitted spacer fabric composites (hereinafter referred to briefly as PWSF, Figure 2) were produced by impregnating these warp-knitted spacer fabrics with a semi-rigid polyurethane foams, consisting of a mix of $1.21 \mathrm{~g} / \mathrm{cm}^{3}$ isocyanate and 0.78 $\mathrm{g} / \mathrm{cm}^{3}$ polyol, in a 43.7/100 isocyanate-polyol mixing ratio (by weight). Production of the composite samples was carried out in a mold whose and bottom surfaces can be heated by a water

Table 1. Chain notations for outer layer structures

\begin{tabular}{|c|c|}
\hline Structure & Chain notation \\
\hline Chain+Inlay & $\begin{array}{l}\text { GB1:0-0 0-0/5-5 5-5// fully threaded GB2:1-0 0-0/1-0 0-0// fully threaded GB5:0-0 1-0/0-0 1-0// fully } \\
\text { threaded GB6:0-0 5-5/5-5 0-0// fully threaded }\end{array}$ \\
\hline Rhombic Mesh & $\begin{array}{l}\text { GB1:1-0 0-0/1-2 2-2/2-3 3-3/2-1 1-1// } 1 \text { fully } 1 \text { empty threaded GB2:2-3 3-3/2-1 1-1/1-0 0-0/1-2 2-2// } 1 \\
\text { fully } 1 \text { empty threaded } \\
\qquad \begin{array}{l}\text { GB5:1-0 1-0/0-0 1-2/2-2 2-3/3-3 2-1// } 1 \text { fully } 1 \text { empty threaded } \\
\text { GB6:2-3 2-3/3-3 2-1/1-1 } 1-0 / 0-0 \text { 1-2// } 1 \text { fully } 1 \text { empty threaded }\end{array}\end{array}$ \\
\hline Hexagonal Mesh & 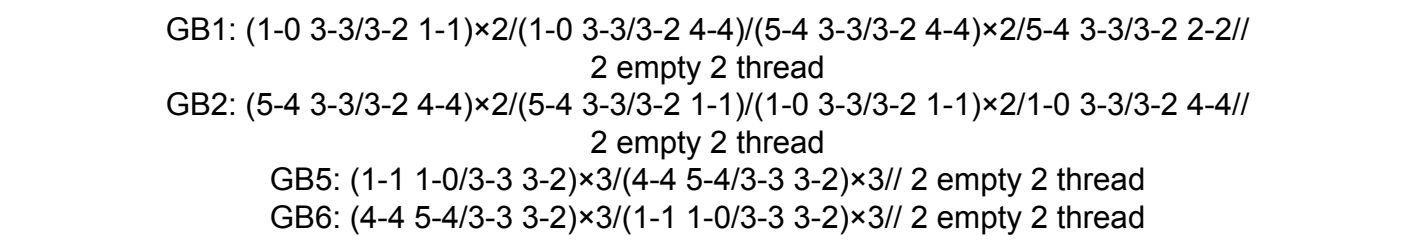 \\
\hline
\end{tabular}

Table 2. Details of spacer yarns

\begin{tabular}{|c|c|c|}
\hline Symbol & Diameter(mm) & \multicolumn{1}{c|}{ Lapping movement } \\
\hline I & 0.2 & $\begin{array}{l}\text { GB3:1-0 3-2/3-2 1-0// } 1 \text { full } 1 \text { empty } \\
\text { GB4:3-2 1-0/1-0 3-2// } 1 \text { empty } 1 \text { full }\end{array}$ \\
\hline II & 0.2 & $\begin{array}{l}\text { GB3:1-0 4-3/4-3 1-0// 1 full 1 empty } \\
\text { GB4:4-3 1-0/1-0 4-3// 1 empty } 1 \text { full }\end{array}$ \\
\hline III & 0.16 & $\begin{array}{l}\text { GB3:1-0 4-3/4-3 1-0// 1 full } 1 \text { empty } \\
\text { GB4:4-3 1-0/1-0 4-3// 1 empty } 1 \text { full }\end{array}$ \\
\hline
\end{tabular}

Table 3. Structural parameters of warp-knitted spacer fabrics

\begin{tabular}{|c|c|c|c|c|c|c|c|}
\hline Specimen & $\begin{array}{c}\text { Thickness } \\
\text { (mm) }\end{array}$ & $\begin{array}{c}\text { Course-wise } \\
\text { density } \\
(w / 5 \mathrm{~cm})\end{array}$ & $\begin{array}{c}\text { Wale-wise } \\
\text { density } \\
(\mathrm{c} / 5 \mathrm{~cm})\end{array}$ & $\begin{array}{l}\text { Area density } \\
\qquad(\mathrm{g} / \mathrm{m} 2)\end{array}$ & Top layer & Spacer yarn & $\begin{array}{c}\text { Bottom } \\
\text { layer }\end{array}$ \\
\hline WSF1 & 7.68 & 34.95 & 28.25 & 911.8 & $\mathrm{C}$ & 1 & $\mathrm{C}$ \\
\hline WSF2 & 7.72 & 35.35 & 27.86 & 881.7 & C & II & C \\
\hline WSF3 & 7.69 & 34.17 & 28.2 & 760.3 & $\mathrm{C}$ & III & $\mathrm{C}$ \\
\hline WSF4 & 6.12 & 33.42 & 27.05 & 820.3 & C & I & C \\
\hline WSF5 & 10.62 & 35.12 & 28.05 & 1027.1 & C & 1 & C \\
\hline \multirow{2}{*}{ WSF6 } & \multirow{2}{*}{7.58} & C: 35.75 & C: 28.50 & \multirow{2}{*}{829.1} & \multirow{2}{*}{ C } & \multirow{2}{*}{ I } & \multirow{2}{*}{$\mathrm{H}$} \\
\hline & & $\mathrm{H}: 36.20$ & $\mathrm{H}: 28.57$ & & & & \\
\hline WSF7 & 7.61 & 34.65 & 27.56 & 756 & $\mathrm{R}$ & I & $\mathrm{H}$ \\
\hline WSF8 & 7.66 & 35.32 & 29.18 & 778.2 & $\mathrm{H}$ & I & $\mathrm{R}$ \\
\hline
\end{tabular}

"C," "H," and "R" represent Chain+Inlay, Hexagonal Mesh, and Rhombic Mesh, respectively. 
bath heating system. Furthermore, the height of the mold can be easily adjusted according to the thickness of corresponding fabrics. The isocyanate-polyol mixing liquid was infused through the core of spacer fabrics in the warp direction. At the same time, the temperature of the water was maintained at $40^{\circ}$ Cduring the infusion process, which was carried out for 8 $\mathrm{min}$, in order to maintain the cured polyurethane foam. After foaming, all the specimens were placed for 24 hours at room temperature, until the polyurethane foam was stable. The eight types of composites produced have been listed in Table 4 .

\subsection{Compression experiments}

The composites were characterized for compression properties according to the Chinese standard GB/T8168-2008 using HuaLong Compression Instrument (Shanghai, China). The diameter of the two compression circular platens is $100 \mathrm{~mm}$. The shape of all the specimens was circle with $60 \mathrm{~mm}$ in

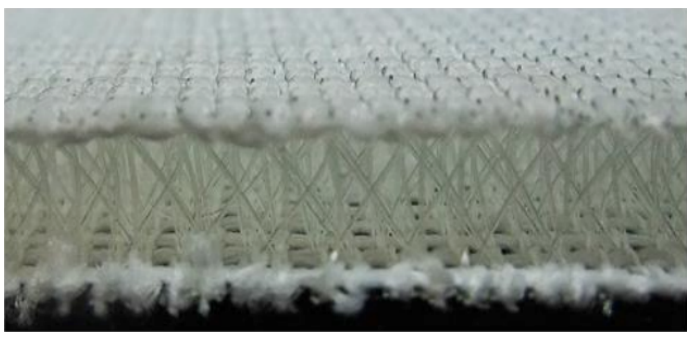

(a) diameter. The specimens were pressed to a deformation with $60 \%$ of the initial thickness at a load speed of $1 \mathrm{~mm} / \mathrm{min}$ in an environment of $23^{\circ} \mathrm{C}$ and $65 \%$ relative humidity. Five repeats were carried out for each specimen. Each compression stressstrain curve provided is an average of the five experimental results.

\section{Result and discussion}

\subsection{The stress-strain curves and energy-absorption efficiency diagrams}

A typical compression stress-strain curve of composite (PWSF1) is shown in Figure 3. According to the published results $[4,10-12]$, the compression stress-strain curve can be split into three stages: linear stage (stage I), plastic plateau stage (stage II), and densification stage (stage III). At the

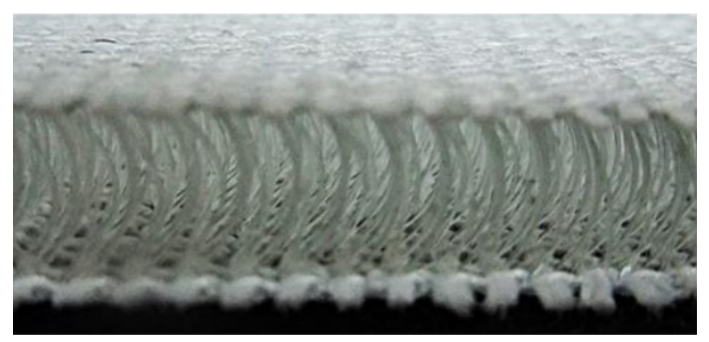

(b)

Figure 1. The front (a) and right (b) view of WSF1

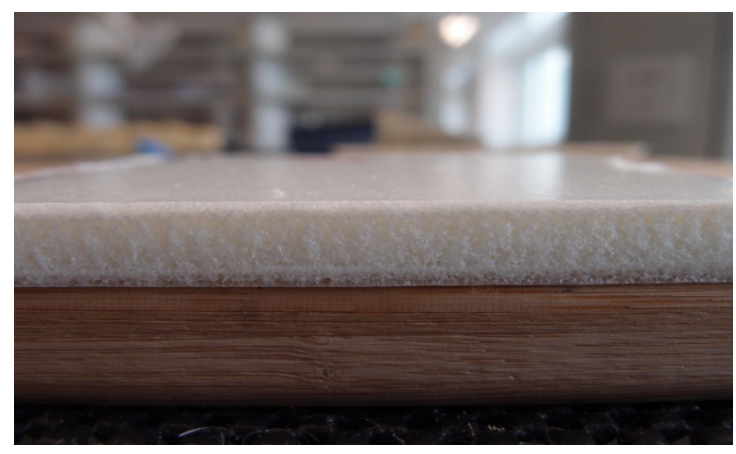

Figure 2. The real appearance of PWSF

Table 4. Details of composites

\begin{tabular}{|c|c|c|c|c|c|}
\hline \multirow{2}{*}{ Sample } & \multirow{2}{*}{$\begin{array}{l}\text { Thickness } \\
\quad(\mathrm{mm})\end{array}$} & \multicolumn{2}{|c|}{ Finished properties of polyurethane foam } & \multirow{2}{*}{$\begin{array}{c}\text { Fiber volume } \\
\text { fraction } \\
(\%)\end{array}$} & \multirow{2}{*}{$\begin{array}{l}\text { Weight ratio of } \\
\text { fabric/foam (\%) }\end{array}$} \\
\hline & & Density (g/cm3) & $\begin{array}{l}\text { Elastic modulus } \\
\text { (MPa) }\end{array}$ & & \\
\hline PWSF1 & 7.68 & 0.795 & 12.09 & 7.33 & $18.2 / 81.8$ \\
\hline PWSF2 & 7.72 & 0.795 & 12.09 & 6.57 & $17.3 / 82.7$ \\
\hline PWSF3 & 7.59 & 0.795 & 12.09 & 6.32 & $17.8 / 82.2$ \\
\hline PWSF4 & 6.12 & 0.795 & 12.09 & 8.91 & $16.8 / 83.2$ \\
\hline PWSF5 & 10.62 & 0.795 & 12.09 & 7.26 & $19.2 / 80.8$ \\
\hline PWSF6 & 7.58 & 0.795 & 12.09 & 6.92 & $17.9 / 82.1$ \\
\hline PWSF7 & 7.61 & 0.795 & 12.09 & 2.25 & $18.3 / 81.7$ \\
\hline PWSF8 & 7.66 & 0.795 & 12.09 & 6.64 & $17.6 / 82.4$ \\
\hline
\end{tabular}


initial stage (stage I), the compression curve exhibits a linear behavior because of the compression of loose foams and their poor constraint capacities for fabrics. When the composite is further compressed, the curve goes to a plateau region in which a relatively constant stress is exhibited. Then, there is a rapid increase in the stress values at stage III, because the entire composite becomes densified at this stage.

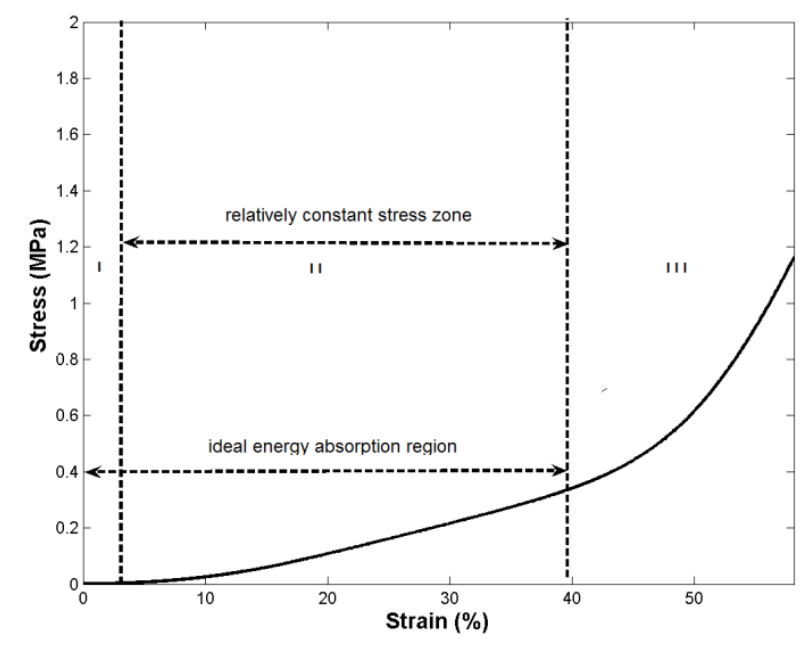

Figure 3. Typical stress-strain curve of composite

Referring to Figure 3, it can be observed that a nearly constant stress is obtained with a large deformation at stage II. This specific behavior is just the requirement of cushioning materials. From the beginning of stage I to the end of stage II, the area under stress-stain curve represents the ideal energy absorbed region where the stress values are low and nearly constant. It is important to point out that at stage III, the energy absorbed by composite is low, but the stress values are high. In these circumstances, cushioning materials should be used to absorb energy before reaching their densification stages in order to avoid high compression stress. The compression stress-strain curves for all the composites are listed in Figure 4.

Although the energy-absorption behaviors can be shown in stress-strain curves, in order to have better view of the energy-absorption process, the energy-absorption efficiency $E$ is involved. The energy-absorption efficiency $E$ is defined as the ratio of the energy absorbed by a real material at a given strain and energy absorbed by an ideal one that transmits the same but constant force at the same strain [13]. The absorption efficiency $E$ can be expressed by Equation (1):

$$
E=\frac{W}{\sigma}=\frac{\int_{0}^{a} \sigma(\varepsilon) d \varepsilon}{\sigma}
$$

where $W$ is the absorbed energy per unit volume and $\sigma$ is the stress at the strain $\varepsilon$.

The energy-absorption efficiency diagrams are shown in Figure 5. From energy-absorption efficiency diagrams, it is clearly observed that a similar variation trend as that of stress-strain curves is exhibited. Furthermore, the maximum efficiency points that can be regarded as the critical points between stage II and stage III are obtained at the end stage II. After this point, the efficiency begins to decrease because of the swift densification of composites. According to the above analysis, it is demonstrated that the efficiency diagrams are useful tools to determinate the preferable energy-absorption region of composites. Therefore, the energy-absorption efficiency diagrams will be used throughout this study to discuss the effect of fabric structural parameters on the compression behaviors of composites.

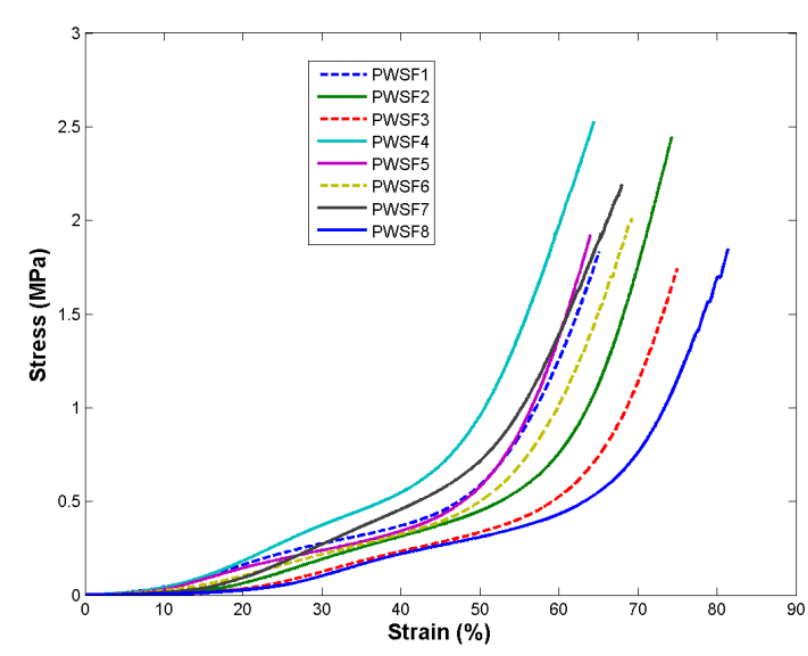

Figure 4. The compression stress-strain curves of composites

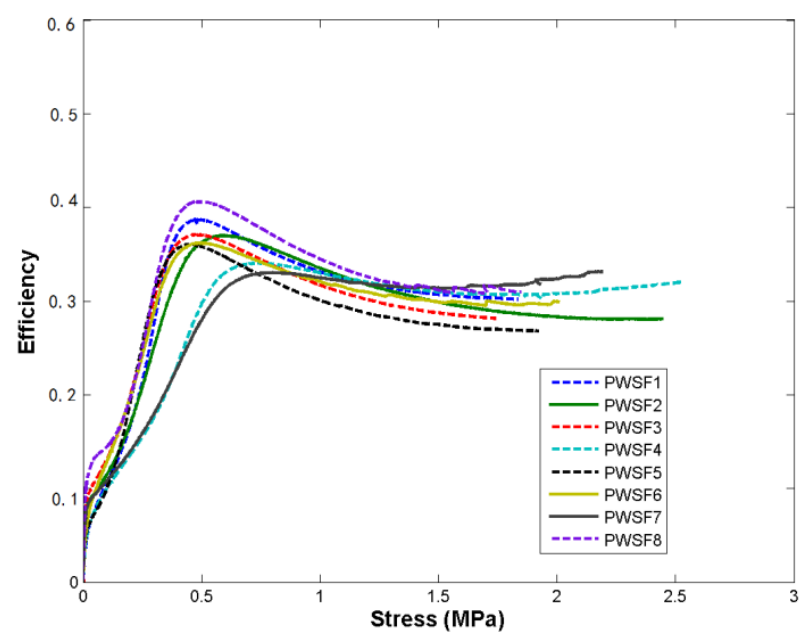

Figure 5. The energy-absorption efficiency diagrams of composites

\subsection{The influence of fabric structural parameters on compression behaviors}

Inclination angle of spacer yarn

Spacer yarns are used to connect two fabric outer layers. The inclination angle (a, Figure 6 ) is defined as the angle between spacer yarns and outer layers. In order to investigate the effect of inclination angle on the compression behaviors of composites, two specimens (PWSF1 and PWSF2) with different inclination angles were used for comparison study.

Figure 6 shows the global arrangements of spacer yarns of type I and II. In Figure 6, the X-, Y-, and Z-axes indicate 
the direction of weft, wale, and thickness of spacer fabric, respectively. All the dots in the figure represent the junctions between spacer yarns and fabric outer layers. The red lines and green lines represent the spacer yarns carried by GB3 and GB4, respectively. In order to have a better view of spacer yarns' arrangements, three adjacent courses are put in three planes: the white dots, representing the first course, are placed in the first plane, while the black and blue dots, representing the second and third courses, respectively, are placed in the subsequent two planes. However, it is necessary to point out that the blue dots placed in the third plane represent the beginning of a new cycle process.

Obviously, it can be found from Figure 6 that the inclination angle decreases as the lapping movement of guide bars (GB3 and GB4) increases. The sequence of inclination angle for these two specimens is PWSF1 > PWSF2.

If the performances of PWSF1 and PWSF2 under compressive loading are compared, it is clear from Figure 4 that the compression resistance increases as the inclination angle increases. The specimen made from larger inclination angle has higher plateau values than the specimen with smaller inclination angle. It is indicated that for a given amount of energy to be absorbed, the specimen with larger inclination angle will have higher stress values because of its higher plateau values. From Figure 5, it can be found that the maximum efficiency decreases as the inclination angle decreases. At lower stress level, less than about $0.3 \mathrm{MPa}$, it can be clearly observed that PWSF2 has higher efficiency. However, after this stress level, its efficiency reverses. The behavior of PWSF1 is the inverse of PWSF2. These results show that at lower stress level, the specimen made from smaller inclination angle has better performance on the energy-absorption capacities. In contrast, the specimen constructed with larger inclination angle is more appropriate for absorbing energy at higher stress level.

\section{Thickness}

Three specimens (PWSF1, PWSF4, and PWSF5) with the same type of spacer yarn and surface layer density were involved in this section. However, the thickness of these specimens is different.

It is interesting to note that PWSF4 has the highest cushioning performance (Figure 4). For PWSF1 and PWSF5, at linear and plateau stages, the compression resistance decreases as the thickness increases; after the strain reaches about $50 \%$, the situation is inversed. At the end of plateau stage, the stress values of PWSF1 drops obviously, while the stress value of PWSF5 has a slight increase. The efficiency-stress diagrams are shown in Figure 5. It can be found that PWSF1 exhibits the highest efficiency, while the efficiency of PWSF4 is the lowest. However, PWSF4's stress values at maximum efficiency are the highest, whereas PWSF5 achieves its maximum efficiency at the lowest stress values. PWSF1 has the middle values of stress at its maximum efficiency. In these circumstances, the specimens made by different thickness have their own ranges of applications. It is inappropriate to simply compare the energy-absorption capacities of specimens made with different thickness. It is necessary to take into consideration the amount of energy to be absorbed and the stress level to be allowed when selecting the thickness of specimens. Furthermore, the efficiency diagrams can be a good tool for optimizing the thickness of composites.

\section{Fineness of spacer yarn}

Two specimens (PWSF2 and PWSF3) with the same lapping movement of spacer yarn, but with different spacer yarns' diameters $(0.2 \mathrm{~mm}$ and $0.16 \mathrm{~mm})$, were involved in this regard. These two specimens also have the similar thickness and outer layer density.

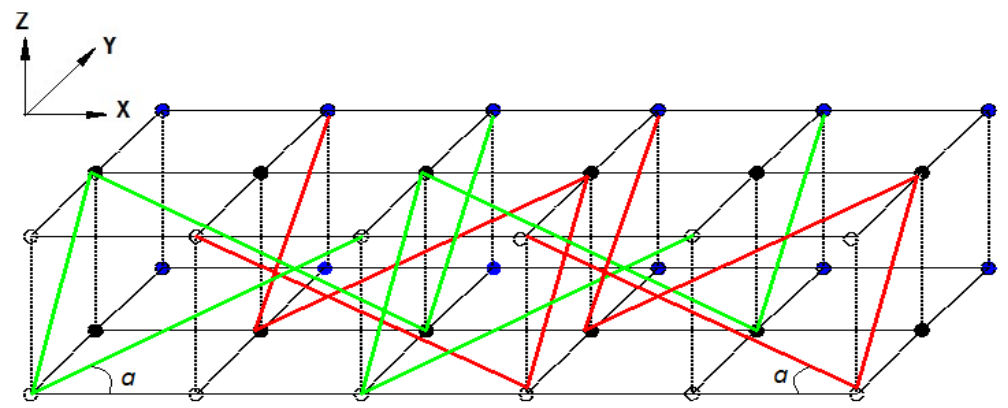

(a)

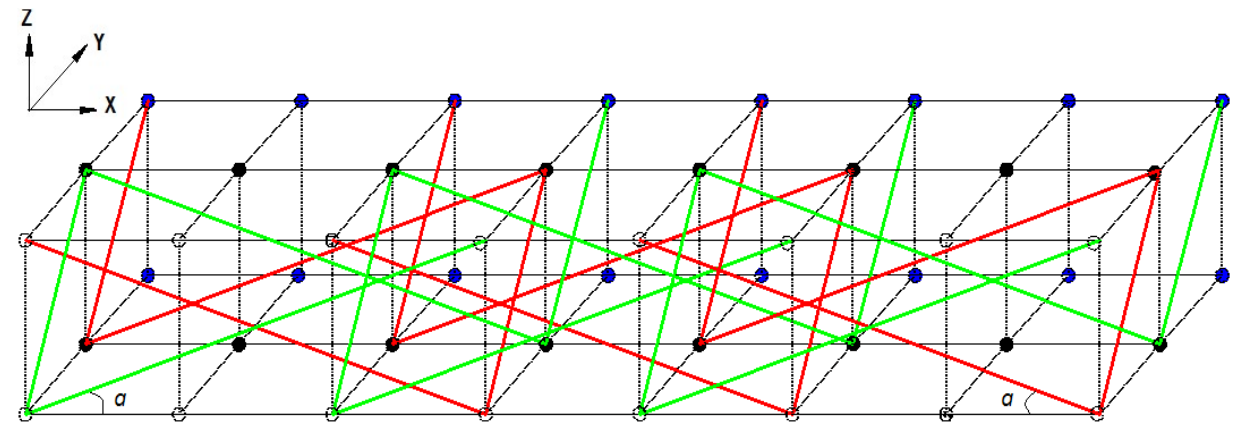

(b)

Figure 6. The global arrangements of spacer yarns: (a) type I and (b) type II 
From Figure 4, it can be seen that the specimen made from coarser spacer yarn has better cushioning performances and higher plateau values. The maximum efficiency values increase with the increasing spacer yarns' diameter, as shown in Figure 5. The stress values at maximum efficiency point are slightly higher for specimen made from coarser spacer yarn. It can also be observed that the specimen constructed of finer spacer yarn has higher efficiency when the stress is less than $0.5 \mathrm{MPa}$, indicating that the specimen made from finer space yarn is suitable for absorbing energy at lower stress level. However, the specimen made from coarser spacer yarn can absorb more energy in a range of stress level higher than 0.5 MPa. Thus, it can be concluded that the specimen made from finer spacer yarn is suitable for lower-stress energy-absorption use, whereas the specimen constructed of coarser spacer yarn can be used for absorbing energy at higher stress level.

\section{Outer layer structure}

In this section, four types of outer layer structures (PWSF1, PWSF6, PWSF7, and PWSF8) were involved for comparison study. These specimens have the same type of spacer yarn and nearly the same thickness. The outer layer structures can be divided into three categories: two-side outer layers with close structure (PWSF1), one-side outer layer with open structure/ one-side surface layer with close structure (PWSF6), and both outer layers with open structures (PWSF7 and PWSF8). Figure 7 shows each of these outer layer structures. It is known that the surface layer structures could slightly influence the outer layer density and spacer yarn's inclination angle, although these parameters were kept constant during the knitting process.

Referring to Figure 4, it is clearly observed that PWSF7 and PWSF8 exhibit the highest and lowest compression resistance, respectively. PWSF1 and PWSF6 have middle compression resistance abilities. The plateau values of PWSF8 are lower than that of the other specimens, which indicates that the maximum efficiency of PWSF8 can be obtained at a lower stress values. On the contrary, PWSF7 will achieve its maximum efficiency at a higher stress level because of its higher plateau values. As shown in Figure 5, it can be revealed that the specimens (PWSF7 and PWSF8) with open structure in two-side outer layers also perform the highest and lowest stress values, respectively, at the maximum efficiency points, whereas the specimens (PWSF6 and PWSF1) with close structure in one-side or both sides surface layers exhibit moderate values. It is implied that the open structures can be used as an energy absorber over a large range of the variations in the stress values. Furthermore, at lower stress level, less than about 1.3 MPa, PWSF8 has the highest energy-absorption efficiency, whereas PWSF7's efficiency is the lowest. However, when the stress is higher than 1.3 $\mathrm{MPa}$, the situation is inversed. The earlier results demonstrate that the specimen made from hexagonal mesh in both surface layers is more suitable for absorbing energy at low stress level, while the specimen constructed of Rhombic Mesh in both outer layers exhibits preferable cushioning behaviors at lower stress values. Thus, varying the structures of outer layers can be another method to adjust the energyabsorption properties of composites.

\section{Conclusions}

In this study, the compression behaviors of WSF composites developed for cushioning applications have been thoroughly investigated. The following conclusions were established through this work:

As a new type of cushioning materials, WSF composites not only have excellent strength but also have outstanding energyabsorption capacities. Furthermore, their energy-absorption capacities can be easily tailored depending on specific enduse requirements by altering the structural parameters of WSF.

The structural parameters of the fabric have obvious influences on the energy-absorption capacities of composites. The specimens with smaller spacer yarn inclination angle, higher fabric thickness, finer spacer yarn, and larger mesh in outer layers are suitable for absorbing energy at lower stress level with higher efficiency. In contrast, the specimens constructed of larger spacer yarn inclination angle, lower fabric thickness, coarser spacer yarn, and smaller mesh in surface layers can be used to absorb energy at higher stress level.

\section{Acknowledgement}

This work was supported by The Foundation of Inner Mongolia University of Technology (ZD201620) and The National Natural Science Foundation of China (11462016).

\section{References}

[1] Lu, Q.S., Sun, L.H., Yang, Z.G., (2010). Optimization on the thermal and tensile influencing factors of polyurethanebased polyester fabric composites, Composites Part A, 41, 997-1005.

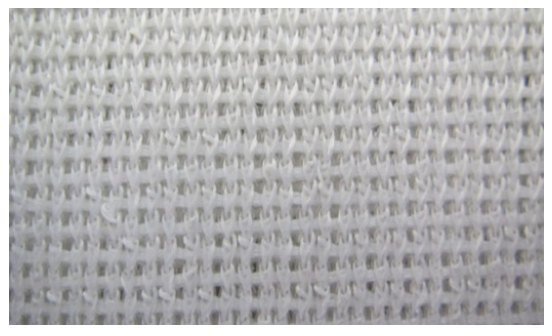

(a)

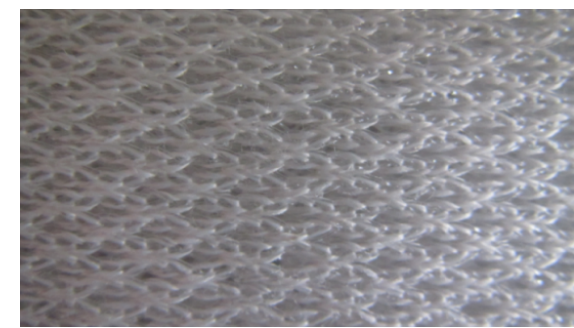

(b)

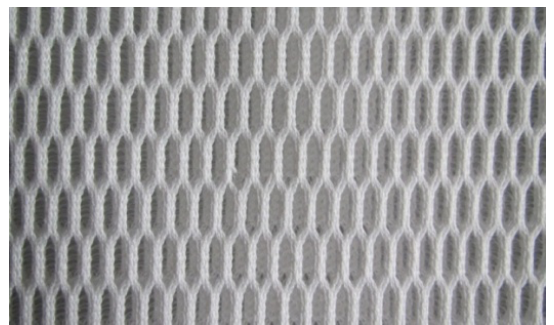

(c)

Figure 7. The surface layer structures: (a) Chain+Inlay, (b) Rhombic Mesh, and (c) Hexagonal Mesh 
[2] Qian, J., Miao, X.H., Shen, Y., (2012). The experimental study and simulation on compressibility of warp-knitted spacer fabrics, Journal of Northwest University, 42(1), 2629.

[3] Janouchova, K., Heller, L. and Vysanska, M., (2013). Functional warp-knitted fabrics with integrated super elastic NITI filaments, Autex Research Journal, 12(3), 3439.

[4] Chen, S., Long, H.R. and Hu, F.C., (2015). Mechanical properties of $3 D$-structure composites based on warpknitted Spacer fabrics, Autex Research Journal, 15(2), 127-137.

[5] Luo, D., I.M., J.J. and Schubel, P.M., (2008). Threedimensional Characterization of Textile Composites, Composites Part B, Vol 39(1), 13-19.

[6] Liu, W.J., Sun, B.Z., Hu, H. and Gu, B.H., (2007). Compressive Behavior of Spacer Weft Knitted Fabric Reinforced Composite at Various Strain Rates, Polymer Composites, 28(2), 224-232.

[7] Dobrich, O., Gereke, T., Cherif, C. (2014). Modelling of Textile Composite Reinforcement on the Micro-scale. Autex Research Journal, 14(1),28-33.
[8] Velosa, J.C., Rana S., Fangueiro R., (2011). Mechanical behavior of novel sandwich composite panels based on 3D-knitted spacer fabrics, Reinforced Plastics and Composites, 31(2), 95-105.

[9] Vuure, van A.W., Pflug, J., Ivens, J.A., Verpoest, I., (2000). Modeling the core properties of composite panels based in woven sandwich-fabrics performs, Composites Science and Technology, 60, 1263-1276.

[10] Lin, Y.L., Lu, F.Y., Wang, X.Y., (2006). Experimental study of the compressible behavior of low-density polyurethane foam, Chinese Journal of High Pressure Physics, 20(1), 89-92.

[11] Miao, X.H. and Ge, M.Q., (2009). Indentation force deflection property of cushioning warp-knitted spacer fabrics, Journal of Textile Research, 30, 43-45.

[12] Mecit, D. and Roye, A., (2009). Investigation of a testing method for compression behavior of spacer fabrics designed for concrete applications, Textile Research Journal, 79, 867-875.

[13] Miltz J., Gruenbaum G., (1981). Evaluation of cushioning properties of plastic foams from compressive measurements, Polymer Engineering Science, 21(2), 1010-1019. 